УДК 536.24:533

\title{
ТЕПЛОМАССООБМЕННЫЕ ПРОЦЕССЫ В ЦИЛИНДРИЧЕСКИХ ГОРЕЛОЧНЫХ УСТРОЙСТВАХ С НИШЕВЫМИ ПОЛОСТЯМИ
}

\author{
Фиалко Н.М., член-корреспондент НАН Украины, Шеренковский Ю.В., канд. техн. наук, \\ Майсон Н.В., Меранова Н.О., канд. техн. наук, Тимощенко А.Б. \\ Институт технической теплофизики НАН Украины, ул. Желябова, 2а, Киев, 03057, Украина
}

Аналізуються результати дос- Анализируются результаты исліджень процесів тепломасопере- следований процессов тепломассопеносу в циліндричних пальниках 3 реноса в цилиндрических горелках кільцевими прямокутними нішовими с кольцевыми прямоугольными нипорожнинами на зовнішній поверхні шевыми полостями на наружной постабілізаторів полум'я. Представле- верхности стабилизаторов пламени. но дані комп'ютерного моделювання Представлены данные компьютернощодо встановлення закономірностей го моделирования по установлению впливу нішової порожнини на ха- закономерностей влияния нишевой рактеристики течії та теплообміну полости на характеристики течения и в пальникових пристроях, що роз- теплообмена в рассматриваемых гоглядаються. Показано, що наявність релочных устройствах. Показано, что ніші може помітно впливати на наличие ниши может заметно влиять інтенсивність процесу горіння, на интенсивность процесса горения, ступінь рівномірності розподілу тем- степень равномерности распределеператури, повноту згоряння палива ния температуры, полноту сгорания тощо.
The results of studies of heat and mass transfer processes in the cylindrical burner with annular rectangular niche cavities on the outside surface of the flame stabilizer are analyzed. The data of computer modeling to establish the patterns of influence of niche cavity on the flow and heat transfer characteristics in considered burners are presented. It is shown that the presence of niches may significantly influence on the intensity of the combustion process, the uniformity degree of the temperature distribution, fullness fuel burning and other parameters.

Библ. 10, табл. 2, рис. 8.

Ключевые слова: цилиндрические горелочные устройства, CFD моделирование, нишевые полости, интенсивность турбулентности, смесеобразование, температурные поля, полнота сгорания топлива.

$d$ - диаметр;

$G$ - расход;

$N_{\Gamma}$ - мощность горелочного устройства;

$I^{T}$ - интенсивность турбулентности;

$P$ - давление;

$r, \varphi, z$ - цилиндрические координаты;

$S$ - шаг расположения газоподающих отверстий;

$T$ - температура;

$U$ - скорость;

$x, \bar{x}$-размерное и безразмерное расстояние от

задней кромки стабилизатора, $\bar{x}=\frac{x}{d \text { ст }}$;

$\gamma$ - коэффициент относительной неравномерности поля температур, $\gamma=\frac{T_{\max }-T_{\mathrm{cp}}}{T_{\mathrm{cp}}-T_{\mathrm{B}}}$; $\eta_{z}$ - коэффициент полноты сгорания топлива;

\section{Сокращения:}

CFD (Computational Fluid Dynamics) - вычислительная гидродинамика;

RANS (Reynolds Averaged Navier-Stokes) методы, базирующиеся на осредненных по Рейнольдсу уравнениях Навье-Стокса;

\section{Индексы:}

0 - начальное значение;

в - воздух;

г- газ;

max - максимальный;

ср - средний;

ст - стабилизатор. 


\section{Введение}

Исследование процессов тепломассопереноса в горелочных устройствах стабилизаторного типа лежит в основе повышения их эффективности и разработки новых технических решений.

В настоящее время все более широкое применение находят цилиндрические стабилизаторные горелки относительно малой мощности. Последние характеризуются легкостью интегрирования в конструкцию энергетического оборудования, низкой металлоемкостью и пр.

Одно из перспективных направлений улучшения технико-экономических характеристик цилиндрических стабилизаторных горелочных устройств связано с использованием различных способов интенсификации процессов тепломассообмена и выгорания топлива в ближнем следе за стабилизатором. Это обуславливает актуальность рассмотрения возможности интенсификации тепломассообменных процессов в данных горелочных устройствах путем применения кольцевых ниш на наружных поверхностях цилиндрических стабилизаторов пламени.

\section{1. Анализ последних исследований и публикаций}

В последний период исследованиям процессов тепломассопереноса в стабилизаторных горелочных устройствах уделяется значительное внимание. Однако в большинстве работ эти исследования относятся к горелочным устройствам с плоскими стабилизаторами пламени (см., например, [1-3]). Что же касается цилиндрических горелочных устройств, то в имеющихся публикациях освещаются лишь некоторые аспекты наблюдаемых в них процессов переноса $[4,5]$.

Выполненный анализ свидетельствует также о том, что в настоящее время все более важным инструментом изучения процессов тепломассопереноса в горелочных устройствах различного типа становится компьютерное моделирование [6-10]. Данное обстоятельство связано, в большой мере, с возможностью получения с использованием компьютерного моделирования локальных характеристик изучаемых процессов, что необходимо для понимания их фундаментальных физических основ и последующей разработки и совершенствования соответствующих техноло- гий и оборудования.

Таким образом, представляет интерес дальнейшее развитие исследований процессов тепломассопереноса в цилиндрических стабилизаторных горелочных устройствах на базе компьютерного моделирования.

\section{2. Формулировка цели статьи}

Целью работы является анализ эффектов влияния кольцевых нишевых полостей на характеристики процессов тепломассопереноса в стабилизаторных цилиндрических горелочных устройствах различной мощности.

\section{3. Особенности математического моделирования}

В работе численные исследования процессов тепломассопереноса в условиях сжигания природного газа проведены для горелочного модуля, представляющего собой круглый канал с соосно расположенным в нем цилиндрическим стабилизатором пламени при наличии кольцевой прямоугольной ниши. Продольный разрез горелочного устройства представлен на рис. 1. Воздух для горения подается на вход цилиндрического канала 1, газообразное топливо через систему круглых отверстий 3, расположенных на боковой поверхности стабилизатора 2 перед нишевой полостью 4, подается внедрением в сносящий поток воздуха.

Математическая постановка рассматриваемой задачи тепломассопереноса имеет вид:

$$
\begin{aligned}
& \frac{\partial}{\partial \tau}(\rho U)+\nabla \cdot(\rho U U)=-\nabla P+\nabla \cdot(S *) \\
& \frac{\partial \rho}{\partial \tau}+\nabla \cdot(\rho U)=0 \\
& \frac{\partial}{\partial \tau}(\rho I)+\nabla \cdot(\rho U I)=\nabla \cdot q_{\Sigma}+q_{V}, \\
& \frac{\partial}{\partial \tau}\left(\rho Y_{i}\right)+\nabla \cdot\left(\rho U Y_{i}\right)=-\nabla \cdot J_{i}+R_{i}, i=1,2, \ldots N-1, \\
& \rho=\frac{P}{R \cdot T \sum_{i}^{N} \frac{Y_{i}}{M_{i}}}
\end{aligned}
$$

где $U$ - вектор скорости; $\tau$ - время; $P$ - статическое давление; $S^{*}$ - тензор напряжений, учиты- 
вающий вязкие напряжения и дополнительные напряжения, обусловленные турбулентностью; $\rho-$ плотность; $q_{\Sigma}-$ суммарный тепловой поток, включающий поток теплопроводностью и дополнительный поток, вызванный турбулентными пульсациями; $q_{V}-$ источниковый член, учитывающий теплоту химических реакций и перенос теплоты радиацией; $I$ - общая энтальпия, $I=\sum_{i} Y_{i} I_{i}$ (здесь $I_{i}, Y_{i}$ - энтальпия и массовая концентрация $i$-го компонента); $R_{i}$ - источниковый член, учитывающий скорость образования $i$-ой компоненты в химической реакции; $J_{i}$ - поток массы $i$-ой компоненты, обусловленный диффузией и турбулентным переносом; $M_{i}$ - молекулярная масса $i$-ой компоненты; $N$ - количество компонент смеси; $T$ - абсолютная температура; $R$ - универсальная газовая постоянная.

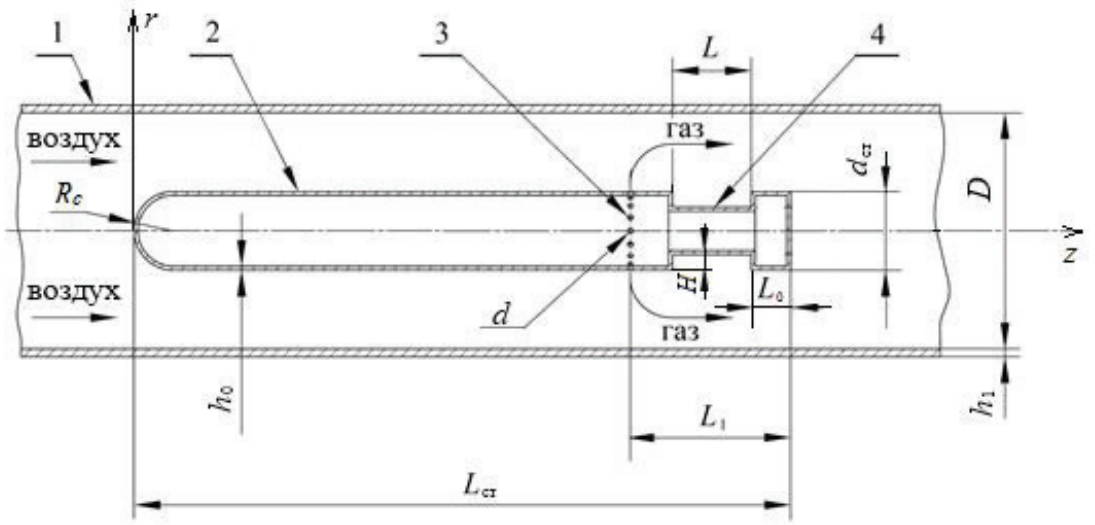

\section{Рис. 1. Продольный разрез горелочного устройства с цилиндрическим стабилизатором пламени: 1 - круглый канал; 2 - цилиндрический стабилизатор пламени; \\ 3 - газоподающие отверстия; 4 -кольцевая ниша.}

Что касается граничных условий для рассматриваемой задачи, то они определялись следующим образом. В сечениях, отвечающих входу в канал горелочного устройства и в газоподающие отверстия, задавались постоянные значения скоростей, концентраций, температур и пр. В выходном сечении канала горелочного устройства ставились так называемые «мягкие» граничные условия - равенство нулю продольных производных всех зависимых переменных. На непроницаемых граничных поверхностях канала и стабилизатора задавались условия прилипания. На боковой поверхности канала принимались условия адиабатичности.

Поставленная задача решалась с использо- ванием RANS подхода и RNG k- $\varepsilon$ модели турбулентности.

Математическое моделирование проводилось при следующих исходных данных: $\alpha=1,1 ; L_{\text {ст }}=0,25 \mathrm{M} ; H=6 \cdot 10^{-3} \mathrm{M} ; h_{0}=1,5 \cdot 10^{-3} \mathrm{M} ;$ $h_{1}=3,0 \cdot 10^{-3} \mathrm{M}$; интенсивность турбулентности воздуха на входе в цилиндрический канал и природного газа в поперечных сечениях газоподающих отверстий $I_{\mathrm{B}}^{\mathrm{T}}=I_{\Gamma}^{\mathrm{T}}=3 \%$; абсолютная температура газа и воздуха на входе 300 К. Рассматривался типоряд горелочных устройств мощностью $N_{\text {г }}=30,110,155$ и 200 кВт. Основные конструктивные и режимные параметры данного типоряда приведены в табл. 1, 2 .

Табл. 1. Основные конструктивные параметры типоряда цилиндрических стабилизаторных горелочных устройств с нишевыми полостями мощностью от 30 до 200 кВт

\begin{tabular}{|c|c|c|c|c|c|c|}
\hline$N_{\mathrm{r}^{\mathrm{r}}} \mathrm{\kappa B \textrm {T }}$ & $d_{\mathrm{cr}} \mathrm{M}$ & $D, \mathrm{M}$ & $d, \mathrm{M}$ & $S / d$ & $L_{0}, \mathrm{M}$ & $L_{1}, \mathrm{M}$ \\
\hline 30 & 0,02 & 0,0365 & 0,002 & 3,5 & 0,015 & 0,049 \\
\hline 110 & 0,04 & 0,073 & 0,003 & 3,22 & 0,025 & 0,062 \\
\hline 155 & 0,05 & 0,091 & 0,0035 & 3 & 0,05 & 0,0885 \\
\hline 200 & 0,06 & 0,1095 & 0,004 & 2,94 & 0,06 & 0,1 \\
\hline
\end{tabular}


Табл. 2. Основные режимные параметры типоряда цилиндрических горелочных устройств мощностью от 30 до 200 кВт в номинальном режиме

\begin{tabular}{|c|c|c|c|c|}
\hline$N$, кBТ & $G, \mathrm{M}^{3} /$ час & $G_{\mathrm{B}}, \mathrm{M}^{3} / \mathrm{час}$ & $U_{r}, \mathrm{M} / \mathrm{c}$ & $U_{\mathrm{B}}, \mathrm{M} / \mathrm{C}$ \\
\hline 30 & 2 & 32,3 & 29,49 & 12,28 \\
\hline 110 & 11 & 118,6 & 33,27 & 11,25 \\
\hline 155 & 15,5 & 167,1 & 31,98 & 10,23 \\
\hline 200 & 20 & 215,6 & 29,49 & 9,09 \\
\hline
\end{tabular}

\section{3. Изложение основного материала}

Ниже представлены характерные результаты компьютерного моделирования процессов переноса в рассматриваемых горелочных устройствах. Рис. 2, 3 иллюстрируют данные о поведении интенсивности турбулентности $I^{\mathrm{T}}$ в цилиндрических горелках мощностью $N_{\Gamma}=110$ кВт с нишевой полостью и при ее отсутствии.

Как показали результаты исследований, закономерности изменения интенсивности турбулентности в горелочных устройствах в условиях наличия и отсутствия ниши могут существенно отличаться. Так, при отсутствии нишевой полости (в сечениях $z=0,209$ м; 0,214 м; 0,220 м) радиальные распределения интенсивности $I^{\mathrm{T}}$ характеризуются наличием двух локальных максимумов (см. рис.3). Первый из них расположен вблизи стенки канала горелки и связан с пристеночной турбулентностью, другой, более ярко выраженный, отвечает оси газовой струи в сносящем потоке окислителя. При наличии же коль- цевой ниши к указанным максимумам добавляется еще один, расположенный в собственно нишевой полости и обусловленный формированием так называемого слоя смешения.

Данные, приведенные на рисунках, свидетельствуют о том, что наличие ниши заметно влияет на величину $I^{\mathrm{T}}$ вблизи наружной поверхности стабилизатора $r=0,02 \mathrm{M}$. С удалением от данной поверхности (с увеличением радиуса $r$ ) это влияние уменьшается, так что на некотором расстоянии от нее становится практически неощутимым. Как видно из рис. 3, чем ближе расположено сечение $z=$ const к входной кромке ниши, тем при меньших значениях $r\left(r=r^{*}\right)$ величины интенсивностей турбулентности становятся практически одинаковыми для ситуаций, отвечающих наличию и отсутствию нишевой полости. Так, при $z=0,209$ м (четверть длины ниши от передней кромки) величина $r^{*}$ равна 0,024 м, а при $z=0,225$ м (задняя стенка ниши) $r^{*}=0,026$. Таким образом, в зоне расположения ниши ее вли-
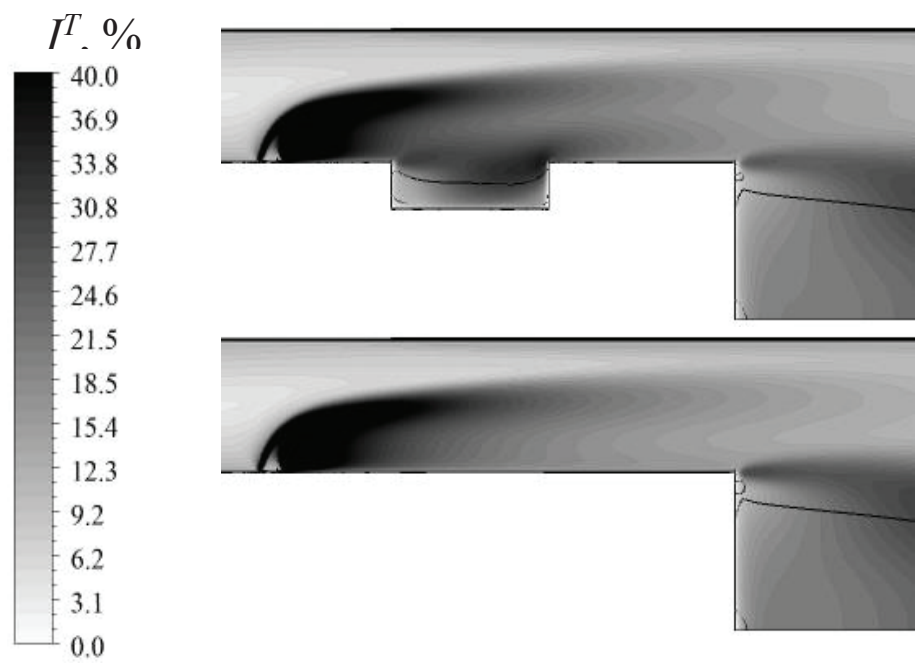

a)

б)

Рис. 2. Поля интенсивности турбулентности в продольном сечении циллиндрической стабилизаторной горелки $\varphi=0$, проходящем через ось газоподающего отверстия, при наличии (а) и отсутствии (б) нищевой полости. 
яние на интенсивность турбулентности потока поверхности стабилизатора. локализуется на расстоянии 4...6 мм от наружной

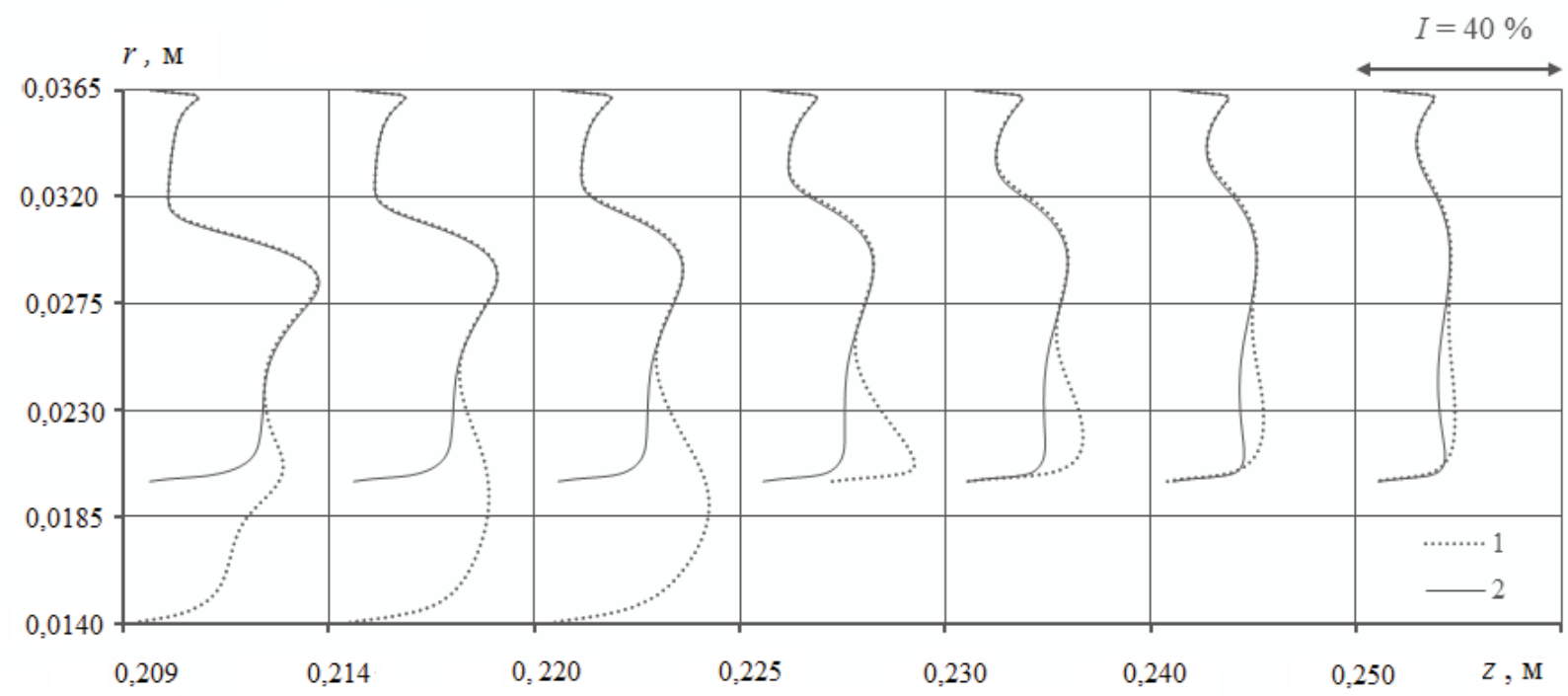

Рис. 3. Распределение интенсивности турбулентности по радиусу горелки мощностью $N_{\Gamma}=110$ кВт при отсутствии (1) и наличии (2) кольцевой ниши для $\varphi=0$ в поперечных

сечениях $z=$ const: $z=0,209$ м (четверть длины ниши); $z=0,214$ м (середина ниши); $z=0,220$ м (три четверти длины ниши); $z=0,225$ м (задняя стенка ниши); $z=0,230$ м; $z=0,240 \mu ; z=0,250$ м (срывная кромка стабилизатора).

Вниз по потоку за нишевой полостью ее влияние на величину $I^{\mathrm{T}}$ охватывает существенно большие области по радиусу канала (рис. 3). Например, на срывной кромке стабилизатора данное влияние ощутимо на расстоянии до 14 мм от наружной поверхности стабилизатора.

Что касается величины отклонений значений интенсивности турбулентности при наличии и отсутствии нишевой полости, то они могут быть значительными. Так, во внешнем потоке в сечении $z=0,225$ м, отвечающем задней стенке ниши, интенсивность турбулентности вблизи наружной поверхности стабилизатора почти вдвое превышает соответствующие значения при отсутствии ниши и составляет $34 \%$.

Следует также отметить, что согласно результатам выполненных исследований потери давления, связанные с наличием кольцевой прямоугольной ниши, незначительны. А именно, для рассматриваемых условий в случае отсутствия ниши потери давления $\Delta P$ в горелочном устройстве равны 61,0 Па, а при ее наличии - 69,0 Па. Таким образом, указанные потери $\Delta P$ увеличиваются только на $13 \%$.
Результаты исследования процессов смесеобразования для рассматриваемых горелочных устройств приведены на рис. 4 и 5 (здесь зоны I характеризуются повышенным содержанием воздуха, при этом массовая концентрация метана меньше нижнего концентрационного предела воспламенения $Y_{\mathrm{CH} 4}<0,028$; зоны II соответствуют повышенному содержанию топлива, где массовая концентрация метана превышает верхний концентрационный предел $Y_{\mathrm{CH} 4}>0,089$; зоны III соответствуют значениям $Y_{\mathrm{CH} 4}$ в концентрационных пределах воспламенения).

Как следует из полученных данных, изменение картины смесеобразования с ростом мощности нишевой горелки сходно с таковыми для случая отсутствия нишевой полости. А именно, с повышением мощности $N_{\Gamma}$ интенсивность смесеобразования в горелочном устройстве снижается. Так, в поперечном сечении, проходящем через заднюю кромку стабилизатора, состояние горючей смеси трансформируется от практически отвечающей концентрационным пределам воспламенения при $N_{\Gamma}=30$ кВт до находящейся в этих пределах лишь в относительно небольшой 
части данного сечения при $N_{\Gamma}=200$ кВт (рис. 4). Однако, при наличии нишевых полостей указанное снижение интенсивности смесеобразования не является столь значительным, как в горелках без таких полостей. Это обусловлено, главным образом, турбулизирующим поток действием собственно ниши и отдалением газоподающих отверстий от срывной кромки стабилизатора в соответствии с требованием организации необходимой картины смесеобразования как в нишевой полости, так и в зоне обратных токов за стабилизатором.

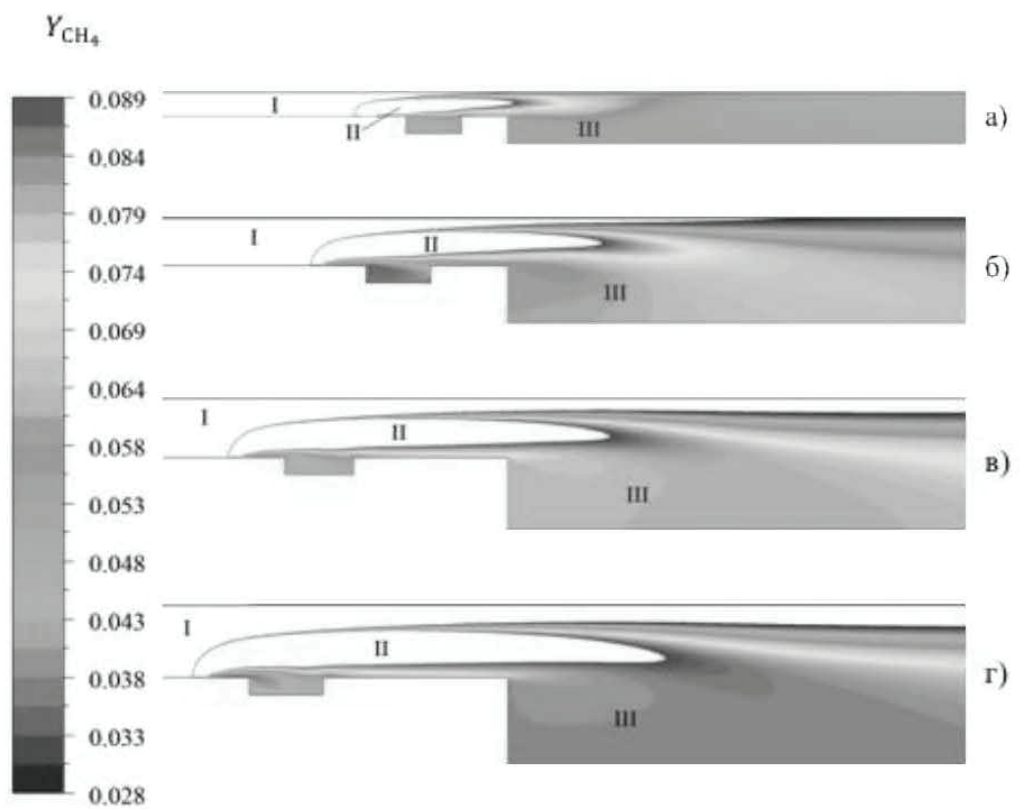

Рис. 4. Поля массовой концентрации метана в продольном сечении цилиндрического горелочного устройства $\varphi=0$, проходящем через ось газоподающего отверстия, при наличии нищевых полостей для горелок разной мощности: a) $N_{\Gamma}=30 \kappa \mathrm{Bm}$; б) $N_{\Gamma}=110 \kappa \mathrm{Bm}$; в) $N_{\Gamma}=155 \kappa \mathrm{Bm}$; г) $N_{\Gamma}=200 \kappa \mathrm{Bm}$.

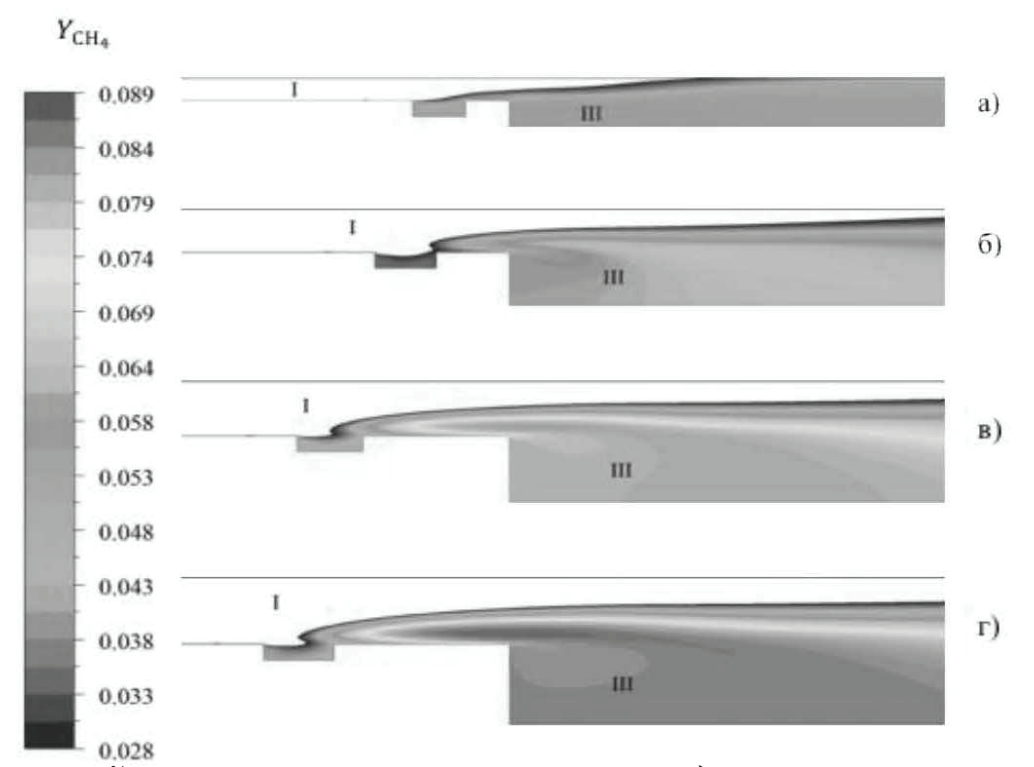

Рис. 5. Поля массовой концентрации метана в продольном сечении цилиндрического горелочного устройства, проходящем посередине между газоподающими отверстиями, при наличии нишевых полостей для горелок разной мощности: a) $N_{\Gamma}=30 \kappa \mathrm{Bm}$; б) $N_{\Gamma}=110 \kappa \mathrm{Bm}$; в) $N_{\Gamma}=155 \kappa \mathrm{Bm}$; г) $N_{\Gamma}=200 \kappa \mathrm{Bm}$. 
На рис. 6 представлены поля температур для цилиндрических горелочных устройств с кольцевой нишей и при её отсутствии. Как видно, в первой из указанных ситуаций горение начинается в собственно нишевой полости, распространяется вдоль наружной поверхности стабилизатора и далее продолжается в его закормовой области. При отсутствии кольцевой ниши начало горения отвечает срывной кромке стабилизатора.

Такой характер процесса горения в сопоставляемых ситуациях обуславливает более высокую интенсивность выгорания топлива и несколько большую равномерность поля температур в поперечных сечениях факела для горелок с кольцевыми прямоугольными нишами (см. рис. 7, 8).

Следует также подчеркнуть, что согласно результатам выполненных исследований, наличие нишевых полостей в цилиндрических горелочных устройствах обусловливает улучшение стабилизации пламени на бедном срыве, что является важным для эксплуатации горелочных устройств на пусковых режимах.
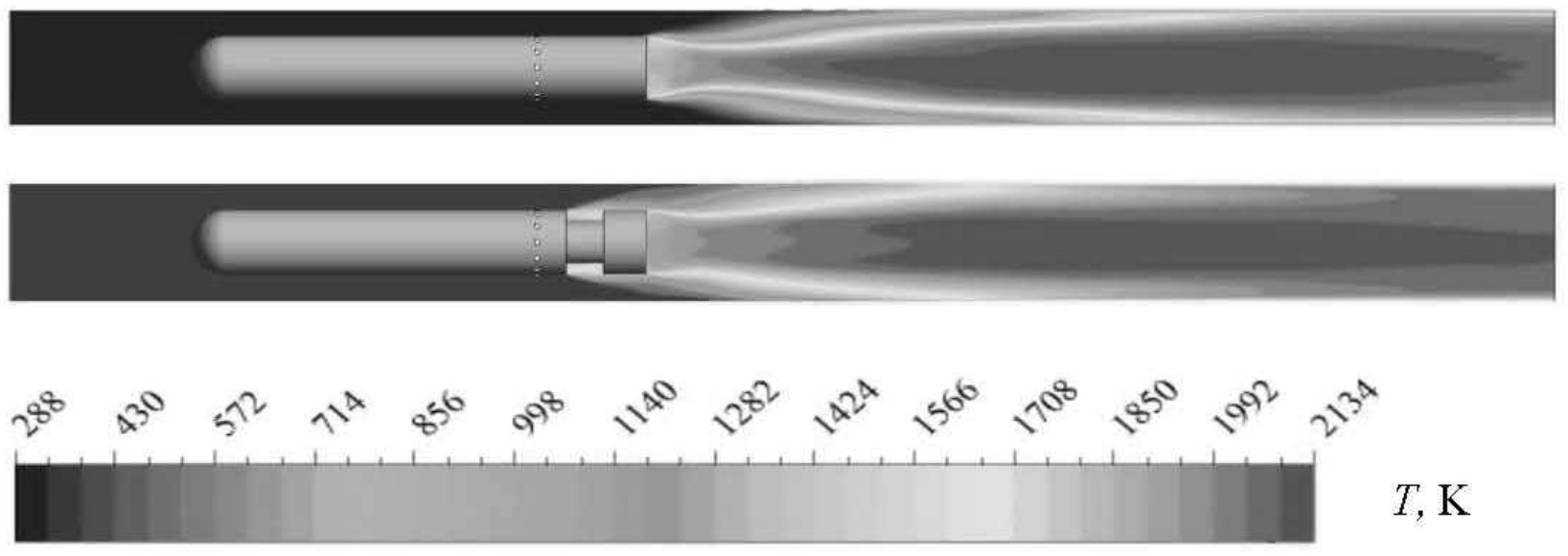

Рис. 6. Поля температур в продольных сечениях, проходящих через ось газоподающего отверстия, для цилиндрического горелочного устройства мощностью $N_{\Gamma}=110 \kappa B m$ при отсутствии (а) и наличии (б) кольцевой прямоугольной ниши.

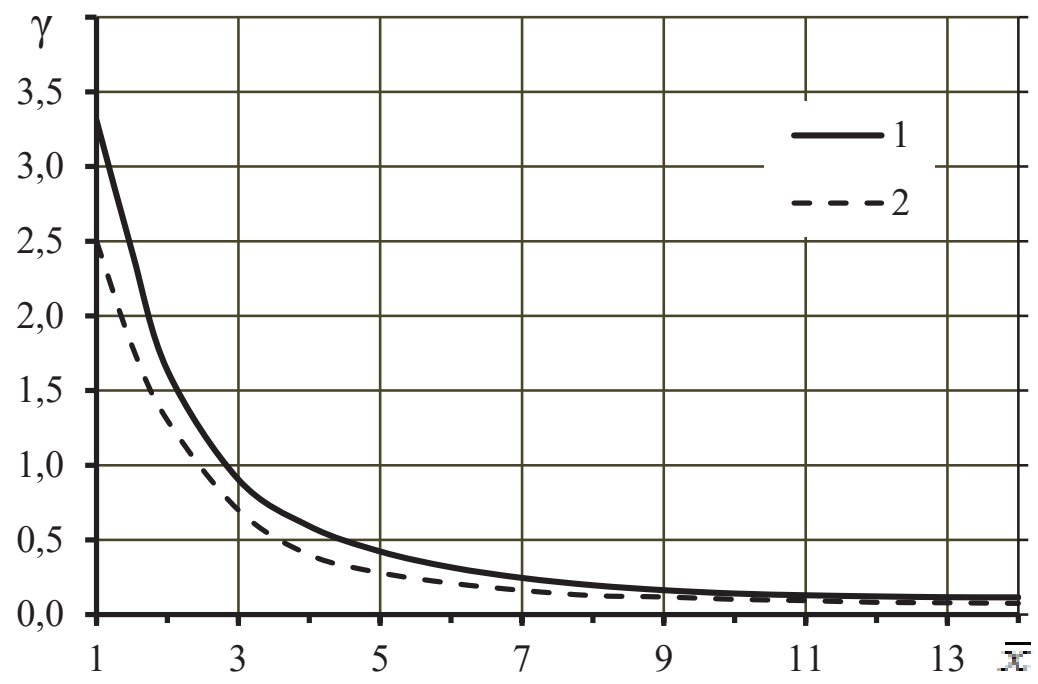

Рис. 7. Относительная неравномерность поля температур у в поперечных сечениях факела для цилиндрического горелочного устройства мощностью $N_{\Gamma}=110 \kappa \mathrm{Bm}$ при отсутствии (1) и наличии (2) кольцевой прямоугольной ниши. 


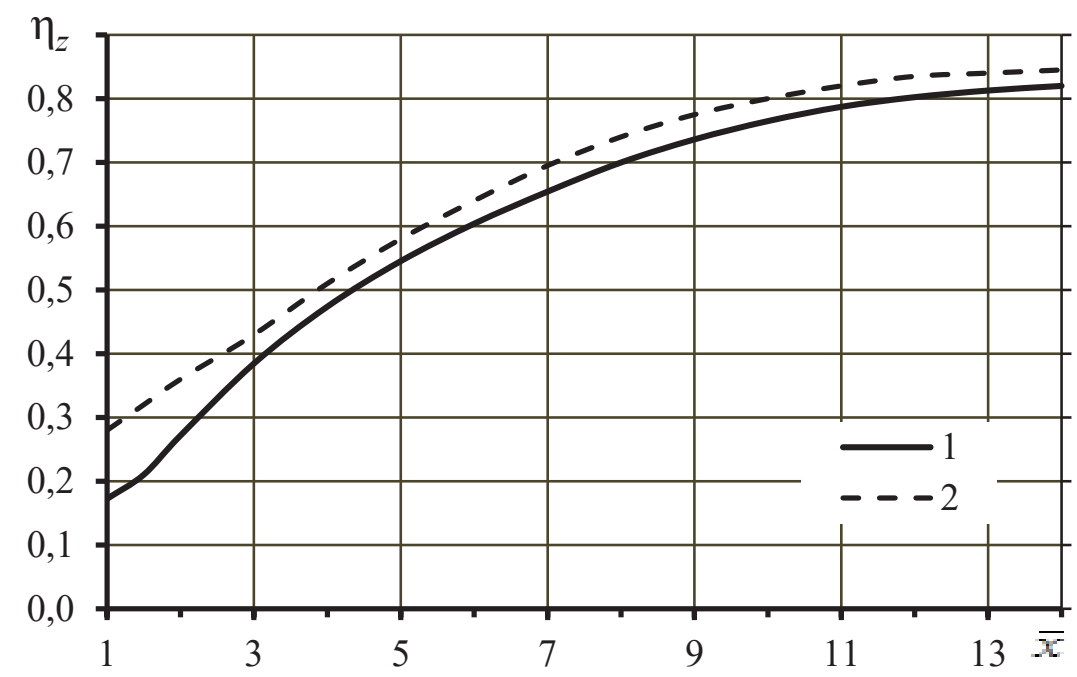

Рис. 8. Изменение коэффициента полноты сгорания топлива по длине факела для цилиндрического горелочного устройства мощностью $N_{\Gamma}=110 \kappa \mathrm{Bm}$ при отсутствии (1) и наличии (2) кольцевой прямоугольной ниши.

\section{Выводы}

1. Выполнены исследования процессов тепломассопереноса для типоряда цилиндрических горелочных устройств мощностью от 30 до 200 кВТ с кольцевыми прямоугольными нишами на наружной поверхности стабилизаторов пламени.

2. Установлены закономерности влияния нишевых полостей на характеристики течения, смесеобразования топлива и окислителя, формирования температурных полей и выгорания топлива в рассматриваемых горелочных устройствах. Показано, что при наличии ниш:

- происходит существенное (до двух раз) повышение интенсивности турбулентности вблизи наружной поверхности стабилизатора пламени и заметное ускорение процесса смесеобразования топлива и окислителя;

- ввиду более раннего по течению начала горения и турбулизирующего поток действия собственно ниши наблюдается более высокая интенсивность выгорания топлива и меньшая степень неравномерности температурных полей;

- потери давления в горелочных устройствах по сравнению с таковыми при отсутствии нишевых полостей повышаются весьма незначительно, не более чем на $13 \%$.

\section{ЛИТЕРАТУРА}

1. Фиалко H.M. Математическое моделирование динамики течения и смесеобразования при сжигании топлива в горелочных устройствах струйно-нишевого типа // Н.М. Фиалко, В.Г. Прокопов, СА. Алешко, Ю.В. Шеренковский, Н.О. Меранова, М.3. Абдулин, Л.С. Бутовский, П.С. Коханенко, Н.П. Полозенко // Пром.теплотехника, 2009, т.31, №7.- С.24.

2. Abdulin M.Z. Research of hydrodynamic flame stabilizer with cross fuel feed characteristics / M.Z. Abdulin, O.A. Siryy // Scientific Journal of Riga Technical University: Series Power and Electrical Engineering. - 2014. - №32. - РP. 12-18.

3. Сірий O.A. Дослідження гідродинаміки потоку повітря в струменево-нишевій системі спалювання палива/ О.А. Сірий, М.З. Абдулін, О.В. Баранюк // Вісник національного технічного університету «ХПІ». - Харків: НТУ «ХПІ», 2016.№9. -С.94-100.

4. Фиалко Н.М. Математическое моделирование процессов течения и смесеобразования в цилиндрическом стабилизаторном горелочном устройстве / Фиалко Н.М., Шеренковский Ю.В., Майсон Н.В., Меранова Н. О., Абдулин М. 3., Бутовский Л. С., Полозенко Н. П., Клищ А. В., Стрижеус С. Н., Тимощенко А. Б. // Восточноевропейский журнал передовых технологий. 2014.- T.3, №8(69). - С. 40-44. 
5. Фиалко Н.М. Интенсификация процессов переноса в горелочном устройстве с цилиндрическим стабилизатором пламени / Фиалко Н.M., Шеренковский Ю.В., Майсон Н.В., Меранова Н. О., Бутовский Л. С., Абдулин М. 3., Полозенко Н. П., Клищ А. В., Стрижеус С. Н., Тимощенко А. Б. // Науковий вісник НЛТУ України. - 2014. - Вип. 24.5 - C. 136-142.

6. Xingsi Han. Simulation of the flame describing function of a turbulent premixed flame using an opensource LES solver/ Xingsi Han , Aimee S. Morgans // Combustion and Flame. - 2015. - V. 162, Issue 5. P. 1778-1792.

7. Hua-Guang Li. A Large-Eddy-Simulation Study of Combustion Dynamics of Bluff-Body Stabilized Flames/ Hua-Guang Li, Prashant Khare, Hong-Gye Sung \& Vigor Yang // Combustion Science and Technology. - 2016. - V. 188, Issue 6. P. 924-952.
8. Ottino G. M. Combustion Modeling Including Heat Loss Using Flamelet Generated Manifolds: A Validation Study in OpenFOAM/ G. M. Ottino , A. Fancello, M. Falcone, R. J. M. Bastiaans, L. P. H. de Goey // Flow, Turbulence and Combustion, April 2016. - V. 96, Issue 3. - P. - 773-800.

9. Bertrand Naud. inklinger RANS modelling of a lifted H2/N2 flame using an unsteady flamelet progress variable approach with presumed PDF / Bertrand Naud, Ricardo Novella, José Manuel Pastor, Johannes F. // Combustion and Flame. - 2015. V. 162, Issue 4. - P. 893-906.

10.Thomas Livebardon. Combining LES of combustion chamber and an actuator disk theory to predict combustion noise in a helicopter engine / Thomas Livebardon, Stéphane Moreau, Laurent Gicquel, Thierry Poinsot, Eric Bouty // Combustion and Flame. - 2016. - V. 165. - P. 272-287. 


\section{HEAT AND MASS TRANSFER PROCESSES IN CYLINDRICAL BURNER DEVICES WITH NICHE CAVITIES}

Fialko N.M., Sherenkovskii Ju.V., Maison N.V., Meranova N.O., Timoshchenko A.B.

Institute of Engineering Thermophysics of National Academy of Sciences of Ukraine, 2a, Zhelyabova str., Kiev, 03057, Ukraine

The results of studies heat and mass transfer processes in the cylindrical burner with annular rectangular niche cavities on the outside surface of the flame stabilizer are analyzed. The features of the flow of these processes for the type series of burners by capacity of $30 \ldots 200 \mathrm{~kW}$ are considered. The data of computer modeling to establish the patterns of influence of niche cavity on the flow and heat transfer characteristics in considered burners are presented. It is shown that the presence of the niche leads to significant turbulence of the flow and accelerate of process of fuel and oxidant mixture formation. It was also established that higher intensity of fuel burn and lower degree of non-uniformity of temperature fields in burning zone are observed in the cylindrical burner devices with niche cavities on the stabilizer surfaces. References 10, figures 8, tables 2.

Key words: Cylindrical burner devices, CFDmodeling, niche cavities, turbulence intensity, mixture formation, temperature fields, fuel burning fullness.

1. Fialko N.M. Matematicheskoe modelirovanie dinamiki techenija I cmeceobrazovanija pri szhiganii topliva $\mathrm{v}$ gorelochnyh ustroistvah strujno-nishevogo tipa [Mathematical modeling of flow dynamics and mixture formation at the burning of fuel in the burner devices of jet-niche type] N.M. Fialko, V.G. Prokopov, S.A. Aleshko, Ju.V. Sherenkovskii, N.O. Meranova, M.Z. Abdulin, L.S. Butovskii, P.S. Kokhanenko, N.P. Polozenko. Promyshlennaja teplotehnika [Industrial Heat Engineering]. 2009, №7. P. 24. (Rus.)

2. Abdulin M.Z. Research of hydrodynamic flame stabilizer with cross fuel feed characteristics . M.Z. Abdulin, O.A. Siryi. Scientific Journal of Riga Technical University: Series Power and Electrical
Engineering. 2014. №32. PP. 12-18.

3. Siryi O.A. Doslidzhennya gidrodinamiki potoku povitrya $\mathrm{V}$ strumenevo-nisheviy sistemi spalyuvannya paliva [Study of air flow hydrodynamic in the jet-niche system of fuel combustion]. O.A. Siryi, M.Z. Abdulin, O.V. Baranyuk. Visnyk natsionalnogo tehnichnogo universitetu "KhPI" [Bulletin of National Technical University "KhPI".]. Kharkiv, NTU "KhPI», 2016. №9. P.94-100. (Ukr.)

4. Fialko N.M. Matematicheskoe modelirovanie processov techenija I smeseobrazovanija v cilindricheskom gorelochnom ustrojstve [Mathematical modeling of processes of flow and mixture formation in the cylindrical stabilizer burner device] N.M. Fialko, Ju.V. Sherenkovskii, N.V. Mayson, N. O. Meranova, M.Z. Abdulin, L.S. Butovskii, H.P. Polozenko, A.V. Klisch, S.N. Strizheus, A.B. Timoshenko. Vostochno Evropejskii zhurnal peredovih technologij [Eastern European journal of advanced technologies]. 2014. Vol.3, №8 (69). P. 40-44. (Rus.)

5. Fialko N.M. Intensifikacija processov perenosa $v$ gorelochnom ustrojstve s cilindricheskim stabilizatorom plameni [Intensification of the transport processes in the burner device with a cylindrical flame stabilizer/ N.M Fialko, J.V. Sherenkovsky, N.V. Mayson, N. O. Meranova, L.S. Butovskii, M.ZAbdulin, N. P. Polozenko, A.V.Klisch, S.N. Strizheus, A.B. Timoshchenko. Naukovii visnik NLTU Ukraini [Scientific Journal NFTU Ukraine]. 2014. Issue 24.5, P. 136-142. (Rus.)

6. Xingsi Han. Simulation of the flame describing function of a turbulent premixed flame using an open-source LES solver. Xingsi Han, Aimee S. Morgans. Combustion and Flame. 2015. V. 162, Issue 5. P. 1778-1792.

7. Hua-Guang Li. A Large-Eddy-Simulation Study of Combustion Dynamics of Bluff-Body Stabilized Flames. Hua-Guang Li, Prashant Khare, Hong-Gye Sung \& Vigor Yang. Combustion Science and Technology. 2016. V. 188, Issue 6. - P. 924-952.

8. Ottino G. M. Combustion Modeling Including Heat Loss Using Flamelet Generated Manifolds: A Validation Study in OpenFOAM. G. M. Ottino, A. Fancello, M. Falcone, R. J. M. Bastiaans, L. P. H. de Goey. Flow, Turbulence and Combustion, April 2016. V. 96, Issue 3. P. 773-800.
9. Bertrand
Naud.
Winklinger
RANS 
modelling of a lifted H2/N2 flame using an unsteady flamelet progress variable approach with presumed PDF. Bertrand Naud, Ricardo Novella, José Manuel Pastor, Johannes F. Combustion and Flame. 2015. V. 162, Issue 4. P. 893-906.

10.Thomas Livebardon. Combining LES of combustion chamber and an actuator disk theory to predict combustion noise in a helicopter engine. Thomas Livebardon, Stéphane Moreau, Laurent Gicquel, Thierry Poinsot, Eric Bouty, Combustion and Flame. 2016. V. 165. P. 272-287.

Получено 15.11.2016 Received 15.11.2016 